\title{
Facectomia na infância: quem são nossos pacientes?
}

\section{Phakectomy in childhood: who are our patients?}

Marcony Rodrigues de Santhiago ${ }^{1}$,Beatriz de Abreu Fiúza Gomes ${ }^{2}$,Newton Kara-José Junior ${ }^{3}$, Noêmia Peixoto da Silva ${ }^{4}$,Carlos Patrício Navarrete ${ }^{5}$, Armando Stefano Crema ${ }^{5}$, Yoshifume Yamane ${ }^{6}$, Sergio Henrique Sampaio Meirelles ${ }^{7}$

\section{Resumo}

Objetivo: Analisar o perfil dos pacientes até 10 anos de idade, submetidos à facectomia no setor de catarata do Hospital da Piedade, determinando as principais indicações, perfil sócioeconômico e características pré-natais nos casos relevantes. Métodos: Foram analisados retrospectivamente, os dados de 60 pacientes com até 10 anos de idade, submetidos à lensectomia, dentro do período de janeiro de 2006 a dezembro de 2007. Todas as cirurgias foram realizadas no setor de catarata do Hospital da Piedade, pelo mesmo cirurgião. Os responsáveis pelas crianças foram submetidos a questionário. Resultados: Dos 60 pacientes, 25 eram do sexo feminino (42\%) e 35 do sexo masculino (58 \%). 42 pacientes (70\%) apresentavam o diagnóstico de catarata congênita, 8 (13\%) tinham subluxação de cristalino e $10(17 \%)$ foram submetidos à lensectomia devido à baixa da acuidade visual associada à catarata traumática. Entre as mães dos pacientes com catarata congênita, $40(95 \%)$ realizaram pré natal com mais de 5 consultas, destas 9 mães $(22,5 \%)$ foram infectadas pelo vírus da rubéola durante a gestação. Nos casos referentes à subluxação todos os 8 apresentavam síndrome de Marfan. Os 10 casos de trauma foram em meninos. Conclusões: Foi possível verificar que a grande maioria de nossos pacientes submetidos à facectomia apresentava catarata congênita e um número relevante dos casos encontrava-se ainda relacionado à rubéola. É fundamental diminuir o tempo entre a suspeita diagnóstica e o atendimento especializado, favorecendo o prognóstico visual dessas crianças.

Descritores: Catarata/congênito; Catarata/diagnóstico; Catarata/epidemiologia; Ambliopia; Estudos retrospectivos

\footnotetext{
'Estagiário do Setor de Catarata e Cirurgia Refrativa do Hospital das Clinicas da Faculdade de Medicina da Universidade de São Paulo - USP - São Paulo (SP), Brasil;

${ }^{2}$ Pós-graduanda (Mestrado) do Serviço de Oftalmologia da Universidade Federal do Rio de Janeiro - UFRJ - Rio de Janeiro (RJ), Brasil;

${ }^{3}$ Livre-docente, Professor Colaborador da Faculdade de Medicina da Universidade de São Paulo - USP - São Paulo (SP), Brasil; Chefe do Setor de Catarata do Hospital das Clinicas da Universidade de São Paulo - USP - São Paulo (SP), Brasil;

${ }^{4}$ Medica Assistente do Setor de Catarata do Hospital da Piedade - Rio de Janeiro (RJ), Brasil;

${ }^{5}$ Chefe do Setor de Catarata do Hospital da Piedade - Rio de Janeiro (RJ), Brasil;

${ }^{6}$ Chefe do Setor de Estrabismo do Hospital da Piedade - Rio de Janeiro (RJ), Brasil;

${ }^{7}$ Chefe do Serviço de Oftalmologia do Hospital da Piedade - Rio de Janeiro (RJ), Brasil.
}

Pesquisa realizada no setor de catarata do Hospital da Piedade - Rio de Janeiro - (RJ ), Brasil.

Recebido para publicação em: 23/9/2008 - Aceito para publicação em 28/7/2009 


\section{INTRODUÇÃO}

D e acordo com a Organização Mundial de Saúde a prevalência de cegueira na infância varia de acordo com as condições de desenvolvimento sócioeconômico e com as taxas de mortalidade infantil dos países. Atualmente estima-se que a catarata pediátrica tratável seja responsável por 10 a $20 \%$ dos casos de cegueira na faixa etária até os $10 \operatorname{anos}^{(1)}$. Representa ainda uma importante parcela das crianças com visão subnormal no Brasil ${ }^{(2)}$.

A cirurgia de catarata na infância continua sendo um desafio para a oftalmologia do terceiro milênio, fazendo-se necessário o seu diagnóstico e tratamento precoce, visando à obtenção de bons resultados funcionais. A recuperação da transparência do eixo visual não é suficiente para a um bom resultado, sendo fundamental o combate à ambliopia por vários anos com equipe multidisciplinar ${ }^{(3)}$.

No Brasil, acomete um grande número de crianças, conforme estudos já realizados, podendo a catarata resultar de malformações oculares congênitas, infecções intrauterinas, síndromes genéticas, alterações sistêmicas com erros inatos do metabolismo, hereditariedade, uso de medicamentos, trauma, radiação ou ser idiopática. ${ }^{(4-6)}$

O objetivo do presente estudo foi analisar o perfil dos pacientes até 10 anos de idade, submetidos à facectomia no setor de catarata do Hospital da Piedade, determinando as principais indicações, perfil sócioeconômico e características pré-natais nos casos relevantes.

\section{Métodos}

O estudo foi realizado após aprovação em comitê de ética e pesquisa do Hospital Municipal da Piedade. Foram analisados retrospectivamente os prontuários de 60 pacientes atendidos no setor de catarata congênita do Hospital da Piedade, entre o período de janeiro de 2006 a outubro de 2007 ,e aplicado questionário estruturado aos 60 genitores. Utilizou-se como critério de inclusão crianças submetidas à facectomia, até 10 anos de idade. Foram excluídos os prontuários que não apresentavam exame diagnóstico preciso ou facectomia em crianças maiores de 10 anos. Investigou-se a indicação da facectomia, sexo, idade ao procurar o serviço oftalmológico, perfil dos pais através de questionário, intercorrências perinatais, infecções maternas durante a gestação, uso de drogas ou fumo durante a gestação, realização de pré-natal, prematuridade, afecções sistêmicas da criança e malformações associadas.

\section{Gráfico 1}

\section{Distribuição da amostra em relação ao sexo - Facectomia na infância no Hospital da Piedade}

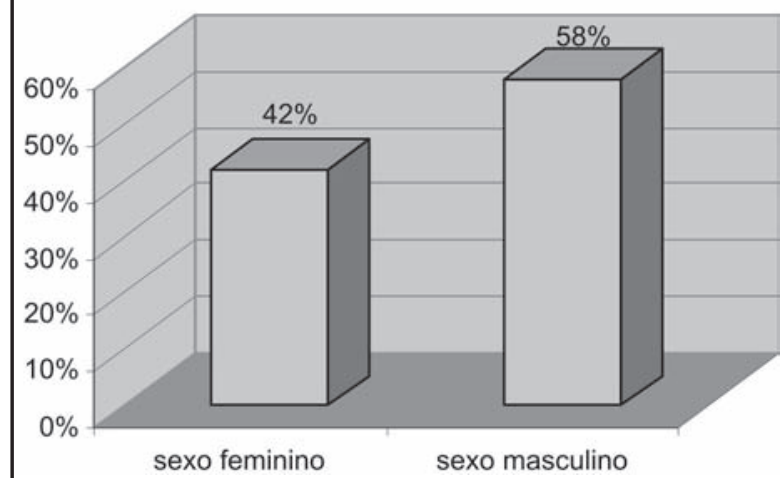

\section{Gráfico 2}

\section{Distribuição da amostra quanto ao diagnóstico pré-operatório - Facectomia na infância no Hospital da Piedade}

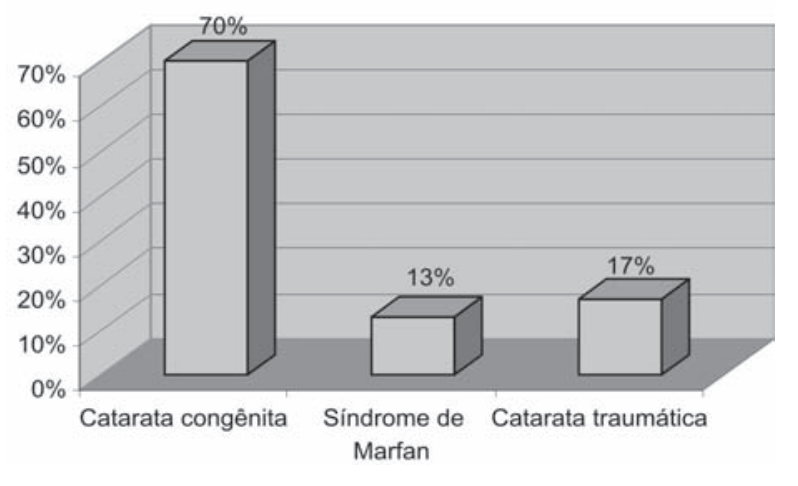

\section{Resultados}

Dos 60 pacientes estudados, 25 eram do sexo feminino e 35 do sexo masculino. (Gráfico 1). A idade das crianças submetidas à lensectomia no momento da pesquisa variou de 0 a 10 anos, com média de 5,7 $\pm 3,3$ anos. Quarenta e dois pacientes (70 \%) apresentavam catarata congênita, 8 (13 \%) subluxação associada à síndrome e $10(17 \%)$ catarata relacionada à trauma. (Gráfico 2).

As oito crianças que apresentavam luxação subtotal do cristalino tiveram diagnóstico de Síndrome de Marfan. Observamos ainda que os 10 casos de catarata relacionada a trauma ocorreram em meninos. 
Nos casos de catarata congênita, a suspeita de alteração ocular, por leigo ou pediatra, ocorreu em $95 \%$ dos casos até os 3 meses de idade. No entanto, a consulta e confirmação diagnóstica ocorreu até os 3 meses em somente $25 \%$ dos pacientes. A maioria dos pacientes foi consultado 3 meses após a suspeita.

Das mães dos pacientes do grupo com catarata congênita (42 crianças), 95 \% (40 mães) realizaram prénatal com mais de cinco consultas. Destas, 9 apresentaram diagnóstico sorológico de infecção por rubéola durante a gestação, 2 casos de toxoplasmose e houve um caso de sífilis. Duas genitoras não realizaram nenhum exame pré-natal.

Quanto ao perfil socioeconômico dos pais de todas as crianças envolvidas no estudo, não apenas as com catarata congênita, verificou-se que a renda familiar na maioria dos casos (78\%) foi de 1 a 3 salários mínimos (Tabela 1).Em relação às genitoras, a maior parte (83\%) apresentava $1^{\circ}$ grau incompleto, enquanto que a maioria dos genitores $(55 \%)$ apresentava $2^{\circ}$ grau incompleto (Tabela 2). Quarenta e seis pacientes (76\%) tinham pais casados, 7 (12\%) viviam com mãe solteira e 7 (12\%) apresentavam pais vivendo em regime de concubinato (Tabela 1). Vinte e cinco mães ( $42 \%$ ) declararam-se fumantes e $10(17 \%)$ disseram ter usado drogas durante a gestação.

\section{DisCUSSÃO}

Do número estimado de 1,4 milhões de crianças cegas no mundo, a catarata é responsável por 190.000 casos e a incidência varia de 1 a 3 para cada 10.000 nascidos vivos ${ }^{(1,7)}$. Associado ao número de cirurgias do cristalino devido a luxação subtotal e trauma, esta conduta ganha participação fundamental no cenário sócioeconômico, principalmente no que se refere aos países em desenvolvimento.

A maioria das lensectomias em crianças realizadas no Hospital da Piedade no período de estudo, foi por conta de catarata congênita. Destes casos, $95 \%$ das mães realizaram pré-natal com mais de 5 consultas e em 9 pacientes $(22,5 \%)$ fez-se diagnóstico sorológico de rubéola e em 2 de toxoplasmose. Os dados do presente estudo foram compatíveis com estudos prévios realizados no Brasil que identificaram a rubéola como causa em $20,9 \%{ }^{(4)}, 14,5 \%{ }^{(5)}$ e $32,5 \%{ }^{(8)}$. Até o desenvolvimento recente da vacina da Rubéola esta representava o fator etiológico em $15 \%$ de todas as cataratas congênitas nos EUA. No Brasil, a rubéola e a toxoplasmose são a primeira e a segunda causa infecciosa de catarata congêni-
Tabela 1

\section{Estado civil e renda familiar dos genitores das crianças submetidas à facectomia no Hospital Municipal da Piedade}

\begin{tabular}{lcc}
\hline Estado civil & n & \% \\
\hline Casado & 46 & 76 \\
Solteiro & 7 & 12 \\
Concubinato & 7 & 12 \\
Renda familiar* & & \\
$<1$ & 6 & 10 \\
$1-3$ & 47 & 78 \\
$>3$ & 7 & 12 \\
\hline
\end{tabular}

* Em salário mínimo

Tabela 2

Grau de instrução dos genitores das crianças submetidos à facectomia no Hospital Municipal da Piedade.

\begin{tabular}{lcccc}
\hline Grau de instrução & \multicolumn{2}{c}{ Mães } & \multicolumn{2}{c}{ Pais } \\
& $\mathbf{n}$ & \% & n & \% \\
\hline Analfabeto & 0 & 0 & 0 & 0 \\
$1^{\circ}$ grau incompleto & 50 & 83,3 & 19 & 31,7 \\
$1^{\circ}$ grau completo & 1 & 1,7 & 2 & 3,3 \\
$2^{\circ}$ grau incompleto & 5 & 8,3 & 33 & 55,0 \\
$2^{\circ}$ grau completo & 2 & 3,3 & 2 & 3,3 \\
Superior incompleto & 1 & 1,7 & 1 & 1,7 \\
Superior completo & 1 & 1,7 & 3 & 5,0 \\
Total & 60 & 100 & 60 & 100 \\
\hline
\end{tabular}

ta, sugerindo falhas na saúde pública do país ${ }^{(3,6)}$.

A maior parte dos genitores do estudo se encontra na faixa que recebe entre 1 e 3 salários mínimos, grande parte com $1^{\circ}$ grau incompleto. Considerando o fato de o Hospital Municipal da Piedade atender prioritariamente a população de baixa renda, parece ser fundamental o estabelecimento de protocolos de "screening" evitando o longo tempo de espera entre a suspeita diagnóstica e o atendimento especializado.

O diagnóstico de catarata congênita é muitas vezes difícil e pode passar despercebido pelos pais ou pelo pediatra, principalmente se não apresentar leucocoria ${ }^{(5)}$. Foi possível identificar que em apenas $25 \%$ de nossos casos o atendimento ocorreu dentro de 3 meses, nos casos de catarata congênita. $\mathrm{O}$ diagnóstico precoce associado à referência ao atendimento especializado seguido de conduta adequada é a maneira mais eficaz de combate ao prognóstico visual desfavorável ${ }^{(9-10)}$. 
O melhor acesso da população aos serviços especializados, além de programas de prevenção realizados em comunidades carentes pode contribuir de maneira significativa na redução da incidência de cegueira irreversível na infância ${ }^{(2,4)}$. Em muitos casos, as crianças já se apresentam, no momento do diagnóstico, com nistagmo, indicando irreversibilidade, de modo que é fundamental estabelecer medidas eficazes para reduzir o tempo entre a suspeita diagnóstica e a primeira consulta ao especialista ${ }^{(10)}$. Atualmente é possível se realizar a facectomia de forma segura na infância com excelente prognóstico visual ${ }^{(1,9)}$.

\section{AbStract}

Objective: To analyze the profile of the patients who were submitted to phakectomy at the cataract service of the Hospital da Piedade, under the age of 10 years old, evaluating the main indications, the socioeconomic and gestational profiles in the relevant cases. Methods: Data of 60 patients under the age of 10 years old submitted to lensectomy, were retrospectively analyzed, during the period between January of 2006 and December of 2007. All the surgeries were performed at the cataract service of the Hospital da Piedade, by the same surgeon. Results: Of the 60 patients, 25 were female (42\%) and 35 were male $(58 \%) .42$ patients $(70 \%)$ had the diagnostic of congenital cataract, $8(13 \%)$ had subluxated lens and 10 (17\%) had lensectomy for traumatic cataract. Considering congenital cataract patient's mothers, 40 (95\%) underwent prenatal exams with more than 5 appointments, $9(22.5 \%)$ of them were infected by rubella virus during the pregnancy. In subluxation cases, 8 had Marfan's syndrome. 10 cases of trauma occurred in boys. Conclusion: It was possible to verify that the majority of our patients submitted to phakectomy had congenital cataract and there is an important number of cases that still associated to rubella. Decreasing time since the suspect until the specialized consultation, will improve the visual prognosis of the children.

Keywords: Cataract/congenital; Cataract/ diagnosis; Cataract/ epidemiology; Amblyopia; Retrospectives studies

\section{RefERÊNCIAS}

1. Gilbert C, Foster A.Childhood blindness in the context of VISION 2020 - the right to sight.Bull World Health Organ. 2001;79(3):227-32.

2. Tartarella MB, Nakano K, Castro CT, Martins AP. Visão subnormal em crianças. Arq Bras Oftalmol. 1991;54(5):221-4.

3. Ventura M. Catarata congênita. In: Rezende F. Cirurgia de catarata. 2a ed. Rio de Janeiro: Cultura Médica; 2002.

4. Oliveira ML, Di Giovanni ME, Porfirio Neto Jr F, Tartarella MB. Catarata congênita: aspectos diagnósticos, clínicos e cirúrgicos em pacientes submetidos a lensectomia. Arq Bras Oftalmol. 2004;67(6):921-6.

5. Adam Netto A, Peres SO. Catarata na infância: estudo de 106 casos. Rev Bras Oftalmol. 1998;57(12):903-8.

6. Kitadai SP, Bonomo PP. Catarata congênita: freqüência etiológica. Arq Bras Oftalmol. 1994;57(6):404-6.

7. Hoyt CS, Good WV. The many challenges of childhood blindness. Br J Ophthalmol. 2001;85(10):1145-6.

8. Cruz CB, Endriss D, Ventura B, Ventura L. Catarata na infância: perfil socioeconômico, gestacional e desenvolvimento neuropsicomotor. Arq Bras Oftalmol. 2005;68(1):9-13.

9. Zetterström C, Kugelberg M. Paediatric cataract surgery. Acta Ophthalmol Scand. 2007;85(7):698-710.

10. Mérula RV, Fernandes LC. Catarata Infantil: importância do diagnóstico e tratamento precoces. Arq Bras Oftalmol. 2005;68(3):299-305.

Endereço para correspondência:

Marcony R. Santhiago

Rua Ataulpho Coutinho, n 200, apt 1202

Condomínio Mandala - Barra da Tijuca

CEP 22793-520 - Rio de Janeiro - RJ - Brasil

E-mail: marconysanthiago@hotmail.com 